\title{
Developmental Regulation of Neuromodulator Function in the Stomatogastric Ganglion of the Lobster, Homarus americanus
}

\author{
Kristina J. Rehm, Katherine E. Deeg, and Eve Marder \\ Volen Center, Brandeis University, Waltham, Massachusetts 02454-9110
}

\begin{abstract}
Neuromodulatory substances have profound effects on the two motor patterns generated by the adult crustacean stomatogastric ganglion (STG), the gastric mill rhythm and the pyloric rhythm. Developmentally regulated changes in the modulatory functions of neuromodulators could therefore play an important role in the maturation of the output from the developing STG. We compared the effects of neuromodulators on isolated embryonic and adult STG of the lobster, Homarus americanus. Bath application of Val ${ }^{1}$-SIFamide, a peptide whose expression is different in embryos and adults, activated different neuron classes in embryos and adults. Cancer borealis tachykinin-related peptide 1a, a peptide that does not appear in the terminals of modulatory neurons in the STG until after embryonic development, also produced different motor patterns in embryos and adults. In contrast, red pigment concentrating hormone, a peptide with a similar distribution in the STNS across development, produced similar motor patterns in embryonic and adult STG. Proctolin, serotonin, and allatostatin were also physiologically active on the isolated embryonic STG. Together, these results demonstrate that receptors to many neuromodulators are present and functional on STG neurons before the motor patterns of the stomatogastric nervous system are mature. Moreover, neuromodulator responses change during development, perhaps contributing to the maturation of the output from the stomatogastric nervous system.
\end{abstract}

Key words: central pattern generator; CabTRP1a; Val ${ }^{1}$-SIFamide; RPCH; crustacean; maturation

\section{Introduction}

Developing neuronal networks are often active before they produce adult behaviors (Feller, 1999; Moody and Bosma, 2005). This early activity is different from adult activity (Wong et al., 1993; Wong and Oakley, 1996; Nishimaru and Kudo, 2000; Demas et al., 2003; Pagliardini et al., 2003; Ren and Greer, 2003; Greer et al., 2006; Warland et al., 2006). Network output maturation could be a result of developmental changes in the underlying network, the neuromodulatory inputs to the network, and/or the effects of these neuromodulators. Studies in vertebrate brain and spinal cord networks have focused on one or several neuromodulators to show that the transition from early spontaneous activity to adult activity approximately coincides with changes in the neuromodulatory inputs and neuromodulatory responses (Rajaofetra et al., 1989; Ziskind-Conhaim et al., 1993; Sillar et al., 1995; Vinay et al., 2000; Rauscent et al., 2006), but

\footnotetext{
Received May 22, 2008; revised July 3, 2008; accepted July 31, 2008.

This work was supported by National Institutes of Health Grants NS050479 (K.J.R.) and NS017813 (E.M.). We thank Dr. Adam L. Taylor at Brandeis University (Waltham, MA) for designing the spectral analysis methods, Dr. Michael P. Nusbaum at the University of Pennsylvania (Philadelphia, PA) for the CabTRP peptide, Dr. Patsy $S$. Dickinson at Bowdoin College (Brunswick, ME) for the Val ${ }^{1}$-SIFamide peptide, and Dr. Andrew E. Christie at the University of Hawaii (Honolulu, HI) for the Val ${ }^{1}$-SIFamide antibody.

Correspondence should be addressed to Kristina J. Rehm at her present address: Dana Farber Cancer Institute, 44 Binney Street, Dana 620, Boston, MA 02115. E-mail: Kristina_Rehm@DFCI.Harvard.edu.

K. E. Deeg's present address: 75 Waterman Street, Box G-LN, Brown University, Providence, RI 02912. D0I:10.1523/JNEUROSCI.2328-08.2008

Copyright $\odot 2008$ Society for Neuroscience $\quad 0270-6474 / 08 / 289828-12 \$ 15.00 / 0$
}

here we provide a more comprehensive study of the responses of a developing nervous system to many neuromodulators.

The adult crustacean stomatogastric nervous system (STNS) is modulated by many different substances (Marder and Bucher, 2007). Our goals were to ask whether multiple neuromodulatory responses are found early in development, to use neuromodulator responses to probe the extent to which the underlying developing networks differ from those in the adult, and to make a developmental comparison of neuromodulator distribution and function.

Motor patterns in the lobster STNS are different in embryos and adults (Casasnovas and Meyrand, 1995; Richards et al., 1999). The adult STNS produces two rhythms, the faster pyloric rhythm $(\sim 1 \mathrm{~Hz})$, and the slower gastric mill rhythm $(\sim 0.1 \mathrm{~Hz})$ (Bucher et al., 2006). In the embryo, neurons that will become pyloric and gastric mill neurons are largely active at a common frequency $(\sim 0.5 \mathrm{~Hz})$ (Casasnovas and Meyrand, 1995; Richards et al., 1999), although late embryonic gastric mill neurons can produce some slow activity (Rehm et al., 2008).

By mid-embryonic development, all stomatogastric ganglion (STG) neurons are present, although little is known about the synaptic connections in the embryonic STG circuit (Casasnovas and Meyrand, 1995; Fénelon et al., 1998; Le Feuvre et al., 1999; Pulver and Marder, 2002). The STG receives input from descending modulatory projection neurons that terminate in the STG neuropil. The complement of neuromodulatory substances in the projection neuron terminals is incomplete until after larval 
development, perhaps contributing to the immaturity of embryonic STNS output (Fénelon et al., 1999; Kilman et al., 1999; Ducret et al., 2007; Cape et al., 2008).

Embryonic and adult STG networks are largely silent when isolated from their modulatory inputs. We elicited motor patterns in embryonic and adult STG by separately bath-applying three modulatory peptides, Cancer borealis tachykinin-related peptide (CabTRP), Val ${ }^{1}$-SIFamide, and red pigment concentrating hormone (RPCH), whose distributions in the STG mature at different rates across development. At a developmental stage when STG network output is immature, the receptors for many neuromodulators are already present, but developmental regulation of the function of a number of these modulators could contribute to the differences between embryonic and adult motor patterns.

\section{Materials and Methods}

Experiments were performed on adult and embryonic lobsters, Homarus americanus. Adult lobsters ( $\sim 500 \mathrm{~g})$ of both sexes were purchased from Yankee Lobster. Egg-bearing females were obtained from the New England Aquarium (Boston, MA) and the Massachusetts State Lobster Hatchery (Martha's Vineyard, MA). All animals were kept in recirculating artificial seawater tanks at $8-14^{\circ} \mathrm{C}$. Eggs were removed from four different females and staged according to Helluy and Beltz (1991). The sex of embryos could not be determined. Embryonic stages are referred to as percentage of total embryonic development (e.g., E80 is $80 \%$ of embryonic development). Embryos were E75-99. (Helluy and Beltz, 1991)

Whole mount immunocytochemistry. Stomachs were dissected out of the embryo and pinned in a Petri dish coated with transparent Sylgard (Dow Corning) using pins cut from 0.0005 gauge tungsten wire (California Fine Wire Company). Most of the brain was removed to allow access to the anterior nerves and ganglia. Stomachs were placed in $4 \%$ paraformaldehyde (Electron Microscopy Sciences) in $0.1 \mathrm{M}$ sodium phosphate buffer, pH 7.3-7.4, overnight at $4^{\circ} \mathrm{C}$. Stomachs were then washed at least six times over $4-8 \mathrm{~h}$ in $0.1 \mathrm{M}$ phosphate buffer containing $0.3 \%$ Triton $\mathrm{X}-100$. The primary antibody was a rabbit anti-Val ${ }^{1}$-SIFamide polyclonal antibody (Christie et al., 2006), donated by Andrew E. Christie (Pacific Biosciences Research Center, University of Hawaii, Honolulu, HI). Christie et al. (2006) used this antibody in the STNS of the adult lobster, Homarus americanus, where they also performed specificity controls. We repeated similar adsorption control experiments, preincubating the primary antibody (1:500) in $10^{-6} \mathrm{M} \mathrm{Val}^{1}$-SIFamide (gift from Patsy S. Dickinson, Department of Biology, Bowdoin College, Brunswick, $\mathrm{ME}$ ) for $2 \mathrm{~h}$ at room temperature before used for primary incubations. Preparations were incubated at $4^{\circ} \mathrm{C}$ for $\sim 72 \mathrm{~h}$ in $1: 500$ rabbit anti-Val ${ }^{1}$-SIFamide primary made in $0.1 \mathrm{M}$ phosphate buffer containing $0.3 \%$ Triton X-100. Preparations were washed at least six times over $4-8$ $\mathrm{h}$ with $0.1 \mathrm{M}$ phosphate buffer. Stomachs were incubated for $12-15 \mathrm{~h}$ at $4^{\circ} \mathrm{C}$ in secondary antibody, goat anti-rabbit IgG conjugated to Alexa Fluor dyes (1:400; Invitrogen) made in $0.1 \mathrm{M}$ phosphate buffer. Stomachs were washed with phosphate buffer at least six times over $6-12 \mathrm{~h}$, dehydrated with an ethanol series $(30,50,70,90$, and 100\%, 5-10 min each), and mounted in methyl salicylate according to Bucher et al. (2007).

Confocal microscopy and image processing. Stomachs were scanned in $1-3$ tiles on $1024 \times 1024$ voxel grids on a Leica TCS confocal microscope using air $(10,20 \times)$ and oil-immersion $(40,63$, and $100 \times)$ objective lenses with z-step sizes of $0.2-0.5 \mu \mathrm{m}$. Image processing, including gray scale adjustment, pixel interpolation, and maximum intensity projection, was performed with AMIRA (version 3.1.1; Mercury). Tiles were concatenated and final figures were created in Canvas (version 10; ADC Systems).

Electrophysiology. Adult lobsters were anesthetized in ice for $\sim 20 \mathrm{~min}$ before dissection, the STNS was dissected from the stomach, pinned in a Petri dish coated with transparent Sylgard, and superfused with chilled $\left(10-15.5^{\circ} \mathrm{C}\right)$ saline. To obtain extracellular recordings, stainless steel pin electrodes were placed in Vaseline wells around the following nerves: pyloric dilator nerve ( $\mathrm{pdn}$ ), lateral pyloric nerve (lpn), and dorsal gastric nerve (dgn). The STG was desheathed and the pyloric dilator (PD), lateral pyloric (LP), and dorsal gastric (DG) motor neurons were identified using 20-40 M $\Omega$ glass microelectrodes, by characteristic waveforms and by matching intracellular spikes with extracellular spikes on the appropriate corresponding nerve. Neuromodulatory inputs were removed in the adult in a two step process. To block action potentials, preventing injury firing and release of neuromodulators, we placed a solution of $10^{-6} \mathrm{M}$ tetrodotoxin (TTX) in $750 \mathrm{~mm}$ sucrose in a Vaseline well around the stomatogastric nerve (stn) directly anterior to the STG. After $30 \mathrm{~min}$ we cut the stn and waited another 30-120 min. The neuromodulatory inputs were considered removed and neuromodulator was applied when the burst frequency of the PD neurons reached steady state.

For embryonic recordings, the whole stomach was removed, and a lateral incision was made up the ventral side of the stomach, preserving the muscles innervated by the PD, LP, and DG neurons. The stomach was pinned flat in a Sylgard coated dish using 0.0005 gauge tungsten wire, and superfused with chilled $\left(11.5-14.5^{\circ} \mathrm{C}\right)$ saline. The embryonic STNS is too small to obtain identified intracellular soma recordings or extracellular nerve recordings. Therefore, glass microelectrodes (20-40 M $\Omega$ ) were used to obtain intracellular recordings of excitatory junctional potentials (EJPs) from muscles innervated by the PD, LP, and DG neurons: PDinnervated muscles (pdm) that will become the cardio-pyloric valve 2 (cpv2) muscles in the adult; LP-innervated muscles (lpm) that will become the cardio-pyloric valve 6 (cpv6) and/or the pyloric 1 (p1) muscles in the adult; DG-innervated muscles (dgm) that will become gastric mill 4 (gm4) muscles in the adult (Richards, 2000). Embryonic neuromodulatory inputs were removed by cutting the stn. All embryonic neurons were silent within 1-2 min of cutting the stn. Neuromodulatory inputs were considered removed and neuromodulator was applied 30-120 min after cutting the stn.

For both adult and embryonic experiments, $10^{-6} \mathrm{M}$ CabTRP1a (amino acid sequence: APSGFLGMRamide; donated by Dr. Michael P. Nusbaum, University of Pennsylvania, Philadelphia, PA; synthesized at the Protein Chemistry Laboratory, University of Pennsylvania, Philadelphia, PA), $10^{-6} \mathrm{M}$ crustacean erythrophore concentrating hormone, or RPCH (amino acid sequence: pELNFSPGWamide; American Peptide Company), $10^{-6} \mathrm{M} \mathrm{Val}^{1}$-SIFamide (amino acid sequence: VYRKPPFNGSIFamide), $10^{-6} \mathrm{M}$ proctolin (Sigma-Aldrich), $10^{-5} \mathrm{M}$ serotonin (5-HT), and $10^{-6} \mathrm{M}$ allatostatin type A 3 (AST-3; Bachem California), $10^{-6} \mathrm{M}$ crustacean cardioactive peptide (CCAP; Bachem California), and $10^{-5} \mathrm{M}$ oxotremorine (Sigma-Aldrich) were bath applied at $\sim 0.05 \mathrm{ml} / \mathrm{s}$. The saline composition for all dissections and experiments was (in mм) $479.12 \mathrm{NaCl}, 12.74 \mathrm{KCl}, 13.67 \mathrm{CaCl}_{2}, 20 \mathrm{Mg}_{2} \mathrm{SO}_{4}, 3.91 \mathrm{Na}_{2} \mathrm{SO}_{4}, 5 \mathrm{HEPES}$, $\mathrm{pH}$ 7.4-7.5. Microelectrodes were filled with $0.6 \mathrm{M} \mathrm{K}_{2} \mathrm{SO}_{4}$ and $20 \mathrm{~mm}$ $\mathrm{KCl}$. Signals were amplified by Axoclamp 2B amplifiers (Axon Instruments), and recordings were digitized using a DigiData 1200 dataacquisition board (Axon Instruments).

Spectral analysis. Physiological recordings were filtered and events were extracted and converted into spike trains in Spike2 (version 5; CED). All spectral analysis was done in Matlab (version 7; The MathWorks) using custom Matlab scripts, according to Rehm et al. (2008). In short, spike trains were converted to a continuous rate-versus-time signal with a Gaussian filter ( $\sigma=50 \mathrm{~ms})$. Spectrograms were created by dividing the signal into temporal windows using multitaper spectral estimation (Percival and Walden, 1993) and by estimating the power spectrum for each window. We used windows of mean length $\mathrm{T} \approx 150 \mathrm{~s}$ ( range $=$ $72-317 \mathrm{~s}$ ), yielding a frequency resolution of $\sim 0.06 \mathrm{~Hz}$. The amount of window overlap was $50 \%$, so that the last half of one window was the same as the first half of the next, resulting in an apparent window length that is half the actual window length.

To estimate the frequency of activity in each neuron, we estimated an overall power spectrum for each spectrogram by averaging the power spectra for every other window (so that the windows were not overlapping). In the power spectra, rhythmic activity appeared as a peak at the fundamental frequency and also as peaks at integer multiples of this frequency called harmonics. By eye we chose the frequency with maximum power density that included the fundamental.

Consistent with Rehm et al. (2008), we used the phase of the peak coherent activity to estimate the relative phase of activity in two neurons. 
First we created coherograms using a multitaper technique similar to that used to create the spectrograms. Coherograms calculate the extent to which the power in two signals is correlated as a function of frequency over time. We then collapsed the coherogram into a single coherence spectrum by averaging the coherence spectra from every other window. From the coherence spectrum, we extracted the coherence magnitude and phase at the frequency of greatest coherence. The coherence magnitude ranges from 0 to 1 , where 0 represents no correlation, and 1 represents perfect correlation at that frequency. Coherence magnitudes were considered statistically significant if they reached the 0.05 level. The coherence phase is given as a fraction of a cycle; therefore a coherence phase of 0 corresponds to synchronous activity, and 0.5 corresponds to alternating activity. We used the coherence phase at the frequency of maximum coherence as the estimate of the phase relationship between two cells.

Statistics. All final figures were created in Canvas (version 10; ADC Systems). Statistical analyses were completed in StatView (version 5; SAS Institute). SD was used to express variability, and error bars were used to represent SEM in the plots of fundamental frequency. Paired $t$ tests were used to compare the mean fundamental frequency of activity in control and neuromodulator conditions; zeros were included in these calculations when the preparations were silent in the absence of modulator. Unpaired $t$ tests and one-way ANOVA tests with Fisher's protected least significant difference (PLSD) post hoc tests were performed to compare fundamental frequencies between two or three cells, respectively. Bonferonni-Dunn post hoc tests were performed when sample sizes were different. Unpaired $t$ tests were also used to compare the mean fundamental frequency of activity in each neuron between embryos and adults. The Watson-Williams test (Igor Pro 6.02A; Wavemetrics) was used to compare the phase of coherent activity between embryos and adults for each cell type.

\section{Results}

\section{Stomatogastric motor patterns of embryos and adults}

When descending modulatory inputs are left intact, both adult and embryonic STG generate rhythmic discharge. However, these rhythms are different in adults and embryos. In all experiments, we used the output from PD and LP motor neurons to monitor pyloric activity, and the output from DG motor neurons as a monitor of gastric mill activity. In adults, we obtained simultaneous intracellular recordings from the somata of the PD, LP, and DG neurons (Fig. 1A). As described by Bucher et al. (2006), bursting was faster in adult PD and LP neurons $(\sim 0.8 \mathrm{~Hz})$ compared with the DG neuron $(\sim 0.1 \mathrm{~Hz})$, although the DG neuron activity also showed simultaneous pyloric-timed activity. Because of the small size of the embryonic nervous system, we monitored motor output from intracellular recordings of the muscles innervated by these neurons (see Materials and Methods). Figure $1 B$ shows simultaneous recordings of a $\mathrm{pdm}, \mathrm{lpm}$, and a dgm in the embryo with neuromodulatory inputs intact. Consistent with previous studies in the embryo (Casasnovas and Meyrand, 1995; Le Feuvre et al., 1999; Rehm et al., 2008), pdm, lpm, and dgm activity occurred at approximately the same frequency $(\sim 0.3$ $\mathrm{Hz}$ ), with slow activity present intermittently in the dgm traces (Fig. $1 B$ ).

\section{Val $^{1}$-SIFamide distribution in the embryonic stomatogastric nervous system}

The differences between adult and embryonic stomatogastric motor patterns could be attributable to developmental differences in the STG network itself, in the synaptic connections or intrinsic membrane properties. Alternatively, these differences could be due to the immaturity of the neuromodulatory inputs (Fénelon et al., 1999; Kilman et al., 1999; Ducret et al., 2007; Cape et al., 2008), or developmental differences in the responses of STG neurons to neuromodulators. Therefore, we asked whether

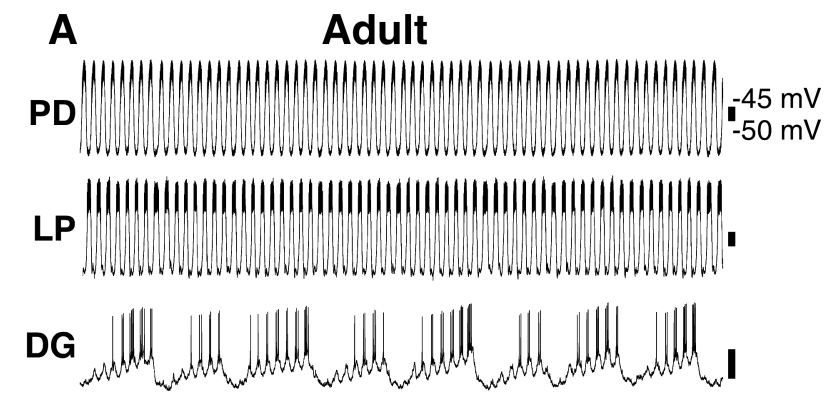

\section{B Embryo}

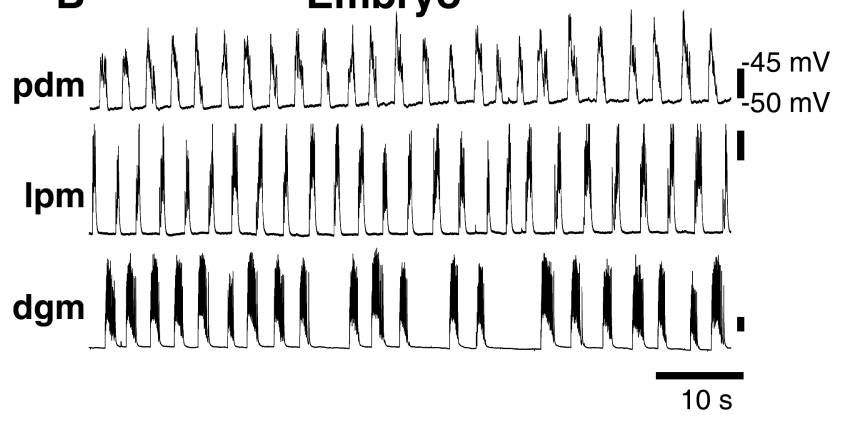

Figure 1. Adult and embryonic stomatogastric motor patterns. A, Adult, Simultaneous intracellular recordings from $P D, L P$, and DG somata, neuromodulatory inputs intact, in control saline. $\boldsymbol{B}$, Embryo, Simultaneous intracellular recordings from pdm, Ipm, and dgm, neuromodulatory inputs intact, in control saline.

$\mathrm{Val}^{1}$-SIFamide, a peptide that can modulate STG neurons in the adult lobster (Christie et al., 2006), was found in the same places in the neuromodulatory inputs of adults and embryos.

Christie et al. (2006) found extensive Val ${ }^{1}$-SIFamide immunolabeling in the neuromodulatory regions of the adult STNS. Using the same antibody and protocols as Christie et al. (2006), we found similar although not identical Val ${ }^{1}$-SIFamide staining in the immature lobster STNS. In the adult, $\mathrm{Val}^{1}$-SIFamide was found in the neuropil regions and in $\sim 45$ somata in the each of the paired commissural ganglia ( $\mathrm{CoG})$, and in two somata in the esophageal ganglion (OG) (Christie et al., 2006). In the CoG of all embryonic preparations $(n=15), \mathrm{Val}^{1}$-SIFamide was found in only one large soma $(\sim 10-15 \mu \mathrm{m})$ with a short process leading to an area of complex branching in the CoG neuropil (Fig. 2A). Although we never saw staining in the embryonic OG, in 3 of 15 preparations we saw one weakly stained fiber directly above the superior esophageal nerve (son)/stn junction leading toward the OG (data not shown). In the adult, $\mathrm{Val}^{1}$-SIFamide was seen in four fasciculated processes in each of the nerves leading to the STG, the two son from each CoG, and the esophageal nerve (on) from the OG, resulting in $12 \mathrm{Val}^{1}$-SIFamide-containing processes in the stn, the nerve that carries the projections of all descending neuromodulatory neurons to the STG (Christie et al., 2006). In contrast, in the embryo we found only one $(n=4)$ (Fig. $2 A, B)$ or two $(n=6)$ (data not shown) $\mathrm{Val}^{1}$-SIFamide-labeled processes in each son, resulting in two to four processes in the embryonic stn (Fig. $2 B$ ). In the adult STG, dense staining in varicosities and branches were seen throughout the peripheral neuropil (Christie et al., 2006). Similarly, in the embryonic STG, dense $\mathrm{Val}^{1}$-SIFamide staining was evident in numerous, diffuse, punctuate varicosities and in the branched neurites of different diameters ranging from $<0.5$ to $2 \mu \mathrm{m}$ (Fig. $2 C$ ). A central core of the embryonic STG was devoid of staining, suggesting that the 

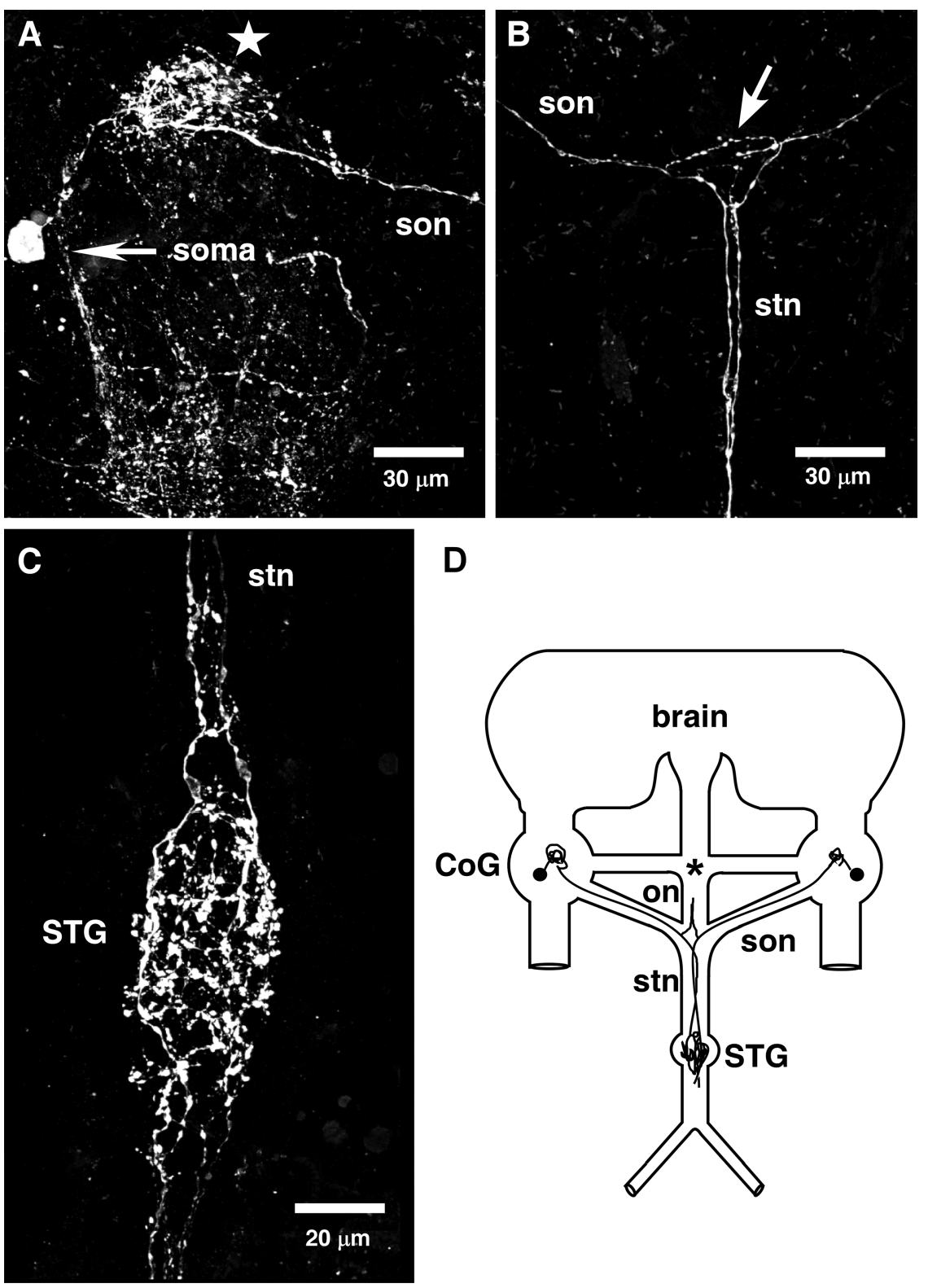

D

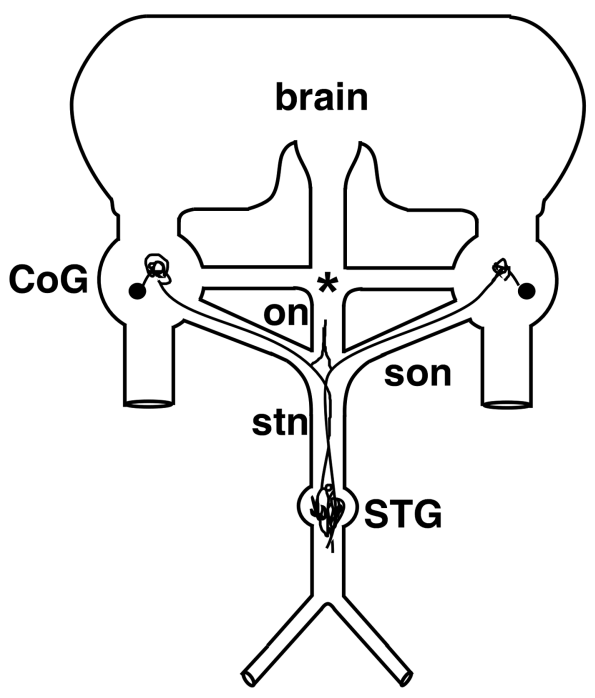

Figure 2. Val ${ }^{1}$-SIFamide distribution in the embryonic stomatogastric nervous system. $\boldsymbol{A}$, Val $^{1}$-SIFamide in the embryonic CoG: one large $(\sim 15 \mu \mathrm{m})$ CoG soma, an arborized region (star), and one process in the son. Image obtained on $63 \times$ lens, 62 optical sections taken at $0.2-0.5 \mu \mathrm{m}$ intervals. $\boldsymbol{B}$, Val ${ }^{1}$-SIFamide in the embryonic son, stn and on junction from same preparation as $A$ : the son process from the $C O G$ in $A$ is found in the upper left-hand corner, leading to the son, on, and stn junction (arrow), meeting the process from the contralateral CoG. Both processes continue down the stn toward the STG. Image was obtained on $63 \times$ lens, 29 optical sections taken at $0.2-0.5 \mu \mathrm{m}$ intervals. C, Val ${ }^{1}$-SIFamide in an embryonic STG from another preparation: extensive Val ${ }^{1}$-SIFamide immunoreactivity is found in round varicosities and axon branches of different diameters ranging from $\sim 0.5-2 \mu \mathrm{m}$. Two anterior tiles were 48 optical sections, bottom tile is 31 optical sections, all taken at $0.2-0.5 \mu \mathrm{m}$ intervals. $\boldsymbol{D}$, Schematic summary of Val ${ }^{1}$-SIFamide distribution in the embryonic stomatogastric nervous system. Val ${ }^{1}$-SIFamide is in one large soma $(\sim 10-15 \mu \mathrm{m})$ and in the CoG neuropil $(n=15)$. One $(n=4)$ or two $(n=6)$ processes were seen in each son in 10 preparations. No staining was evident in the $\mathrm{OG}\left({ }^{*}\right)$, but in 3 of 15 preparations one weakly stained fiber appeared in the lower on. Son processes were also stained in the stn, resulting in two $(n=4)$ or four $(n=6)$ fibers. Extensive staining in the STG was found in 15 embryonic preparations.

$\mathrm{Val}^{1}$-SIFamide staining was limited to the peripheral neuropil (data not shown).

The $\mathrm{Val}^{1}$-SIFamide distribution pattern was not substantially different in the STNS of lobsters at larval stages 1 (L1) and 2 (L2). However, in 6 of 7 L1 preparations and 4 of 5 L2 preparations, two or more fibers were stained for $\mathrm{Val}^{1}$-SIFamide in the son and at least four processes were stained in the stn (data not shown).
The $\mathrm{Val}^{1}$-SIFamide distribution in the embryonic and larval STNS is summarized in the schematic in Figure 2D.

\section{Direct effects of $\mathrm{Val}^{1}$-SIFamide were} different in adult and embryonic STG Although our immunocytochemistry results showed few developmental differences in the $\mathrm{Val}^{1}$-SIFamide distribution, recent matrix-assisted laser desorption/ ionization (MALDI) mass spectrometry experiments showed a developmental difference in the $\mathrm{Val}^{1}$-SIFamide expression: $\mathrm{Val}^{1}$-SIFamide is the most abundant peptide in the embryonic STG, whereas its relative abundance was significantly lower in the adult STG (Cape et al., 2008). This greater relative abundance suggests a more pronounced role for $\mathrm{Val}^{1}$-SIFamide in the embryo. Therefore we wanted to compare the modulatory effects of this peptide in the embryo to those in the adult STG.

Christie et al. (2006) showed the direct effects of $\mathrm{Val}^{1}$-SIFamide on adult pyloric rhythms, however, they did not determine the effects of $\mathrm{Val}^{1}$-SIFamide on adult gastric mill neurons. Therefore, we recorded from the somata of adult PD, LP and DG neurons in the isolated adult STG (after cutting the stn). In control saline, the adult PD neuron fired bursts of action potentials with a burst period of $\sim 15 \mathrm{~s}$, LP was silent, and DG spiked tonically (Fig. 3A). When $\mathrm{Val}^{1}$-SIFamide was bath-applied to this preparation, the PD neuron's burst frequency increased, the LP neuron remained silent, and the DG neuron fired in bursts in which the mean firing rate, at $\sim 0.14 \mathrm{~Hz}$, was coordinated with the PD neuron burst (Fig. 3B).

When the embryonic STG was isolated from its neuromodulatory inputs, all neurons were silent (Fig. 3C). In contrast to the adult, when $\mathrm{Val}^{1}$-SIFamide was bathapplied, all embryonic neurons became active (Fig. $3 D$ ). In this period of recording, bursts of activity in pdm and dgm occurred together and were slower than activity in lpm (Fig. 3D).

Conventional tools for analyzing the rhythmic activity in STG neurons rely on activity being composed of discrete bursts of action potentials. However, the activity we observed did not always have welldefined bursts (Fig. 3D) (e.g., embryonic lpm activity in $\mathrm{Val}^{1}$-SIFamide). Therefore, we used spectral analysis to determine the frequencies of activity ( $y$-axis) over time ( $x$-axis). Figure 4 shows the spectrograms of the entire recording during $\mathrm{Val}^{1}$-SIFamide application in the adult and the embryo shown in Figure 3. In these spectrograms, power is represented by color, where colors toward the blue end of the spectrum indicate low power, and colors toward the red end of the spectrum indicate high power. The fundamental fre- 
quency, the lowest band of activity (see Materials and Methods), in the spectrograms of adult PD and DG activity in $\mathrm{Val}^{1}$ SIFamide was found at $\sim 0.1 \mathrm{~Hz}$, although activity was found at a wider range of frequencies in DG (Fig. 4A). In the embryonic spectrograms, the fundamental frequency of pdm and dgm activity in Val ${ }^{1}$-SIFamide was at $\sim 0.06 \mathrm{~Hz}$ and at $\sim 0.2-0.5 \mathrm{~Hz}$ in lpm (Fig. $4 B$ ).

To examine the modulatory responses to $\mathrm{Val}^{1}$-SIFamide across all preparations, we used the fundamental frequency to calculate the frequency at which most of the activity occurred in each cell type (see Materials and Methods). When the neuromodulatory inputs were removed, adult LP neurons were silent both in control saline $(n=5)$ (data not shown) and in $\mathrm{Val}^{1}$ SIFamide $(n=5)$ (Fig. $5 A)$. Although in some preparations (Fig. $3 A, B), \mathrm{Val}^{1}$ SIFamide appeared to increase the burst frequency in PD neurons compared with control saline, this effect was not significant across preparations (mean fundamental frequencies: in control saline, $0.10 \pm 0.07 \mathrm{~Hz}, n=5$; in $\mathrm{Val}^{1}$-SIFamide, $0.12 \pm 0.06 \mathrm{~Hz}, n=5$; paired $t$ test, $p=$ $0.71, n=5$ ) (Fig. $5 A$, control saline data not shown). Similarly, the mean fundamental frequency of activity in DG neurons in $\mathrm{Val}^{1}$-SIFamide was not significantly different from the mean fundamental frequency of firing in DG neurons in control saline (mean fundamental frequencies: in control saline, $0.47 \pm 0.26 \mathrm{~Hz}, n=5$; in $\mathrm{Val}^{1}$-SIFamide, $0.22 \pm 0.23 \mathrm{~Hz}, n=5$; paired $t$ test, $p=$ $0.24, n=5$ ) (Fig. $5 A$, control saline data not shown). The mean fundamental frequencies of $\mathrm{Val}^{1}$-SIFamide-elicited activity in adult DG and PD neurons were not significantly different (unpaired $t$ test, $p=$ $0.31, n=5)$.

In embryos, when the neuromodulatory inputs were removed, all neurons were silent (Fig. 3C). In $\mathrm{Val}^{1}$-SIFamide, embryonic pdm and dgm, and lpm were activated (Fig. 5B). This activity consisted of excitatory junctional potentials (EJPs) resulting from neurotransmitter released from motor neurons and not direct excitation of the muscles, because $\mathrm{Val}^{1}$-SIFamide-induced activity was not affected by hyperpolarization of the muscles, and the waveforms of the events were typical of nerve-evoked EJPs. The mean fundamental frequencies of activity in embryonic pdm $(0.09 \pm 0.03 \mathrm{~Hz}, n=6)$ and $\operatorname{dgm}(0.06 \pm 0.03 \mathrm{~Hz}, n=7)$ were not significantly different from each other (unpaired $t$ test, $p=0.1$ ), but the mean fundamental frequency of activity in $\operatorname{lpm}(0.33 \pm 0.09 \mathrm{~Hz}, n=6)$ was significantly higher than that of both pdm and dgm [one-way ANOVA, Fisher's PLSD, $p<0.0001$; Bonferroni/Dunn post hoc test, $p<0.0001, n=6$ (lpm, pdm), $n=7$ (dgm)] (Fig. 5B).

When we compared the mean fundamental frequency of ac-
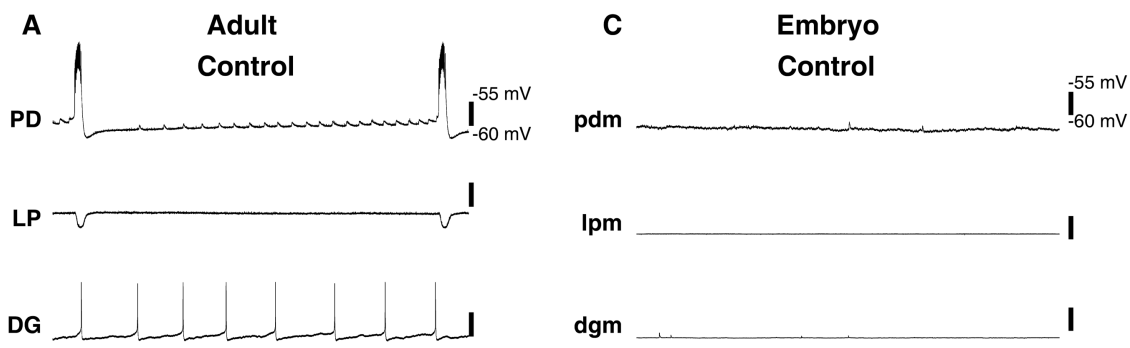

dgm I
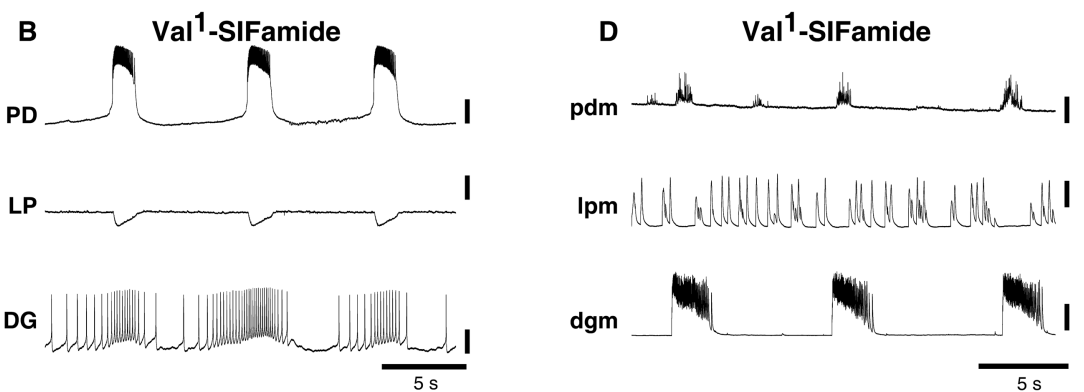

Figure 3. Val ${ }^{1}$-SIFamide effects on an isolated adult and embryonic STG. $\boldsymbol{A}$, Adult, Simultaneous intracellular recordings from $P D, L P$, and DG somata, neuromodulatory inputs removed, in control saline. $\boldsymbol{B}$, Same preparation as in $\boldsymbol{A}$ in $10^{-6} \mathrm{M}$ Val ${ }^{1}$-SIFamide. C, Embryo, Simultaneous intracellular recordings from pdm, Ipm, and dgm, neuromodulatory inputs removed, in control saline. $\boldsymbol{D}$,

A
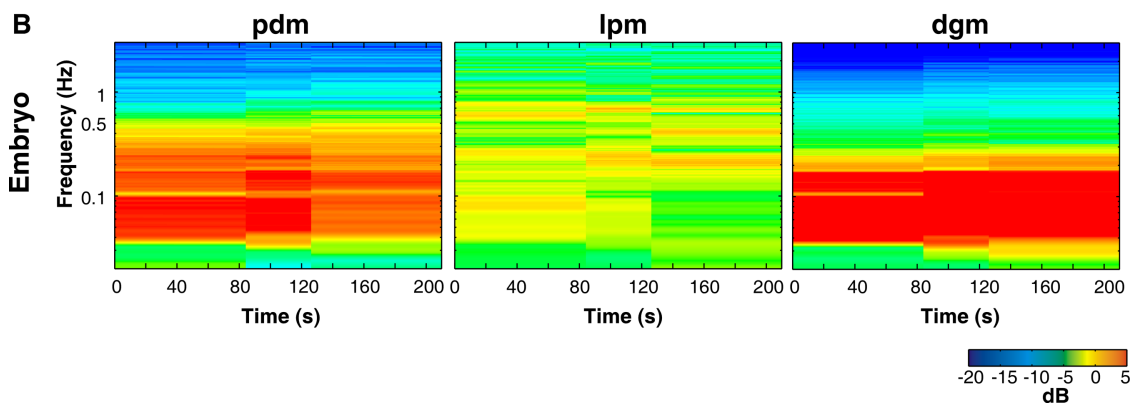

Figure 4. Spectrograms of adult and embryonic Val ${ }^{1}$-SIFamide-activated motor patterns. Spectrograms show power at log scale frequencies in which most predominant frequencies of activity are red and there is no activity at frequencies in dark blue. $\boldsymbol{A}$, Adult PD, LP, and DG spectrograms, total recording time in $10^{-6} \mathrm{M}$ Val ${ }^{1}$-SIFamide from Figure $3 B$. $B$, Embryonic pdm, Ipm, and dgm spectrograms, total recording time in $10^{-6} \mathrm{M}$ Val ${ }^{1}$-SIFamide, preparation shown in Figure $3 D$.

tivity in $\mathrm{Val}^{1}{ }^{1}$-SIFamide across development, the only significant difference was that LP neurons were activated in embryos and not in adults (Fig. $5 A, B$ ). There were no significant differences between the mean fundamental frequencies of activity in adult PD and embryonic pdm (unpaired $t$ test, $p=0.40, n=5$ ). Although the mean fundamental frequency of activity in adult DG neurons tended to be higher than in embryonic dgm, this difference was not statistically significant [unpaired $t$ test, $p=0.08, n=7$ (embryo), $n=5$ (adult)]. These calculations did not include the 3 of 10 embryonic preparations in which dgm was silent or produced only $1-3$ bursts over $\sim 5-10 \mathrm{~min}$.

Neuromodulator-activated motor patterns could also differ 

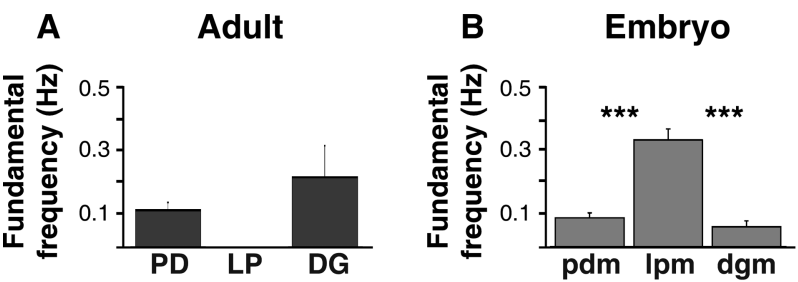

C

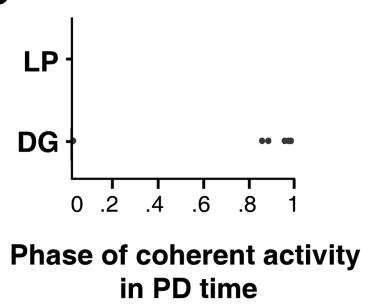

D

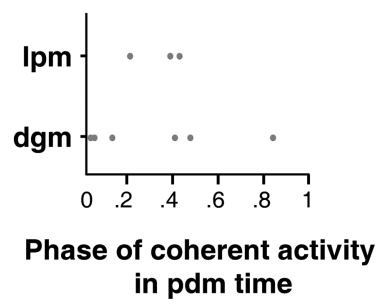

Figure 5. Summary of frequency and phase relationships of adult and embryonic Val ${ }^{1}$. SIFamide-activated motor patterns. $\boldsymbol{A}-\boldsymbol{C}$, Output from adult $(\boldsymbol{A}, \boldsymbol{C})$ and embryonic $(\boldsymbol{B}, \boldsymbol{D})$ neurons during $10^{-6} \mathrm{M} \mathrm{Val}{ }^{1}$-SIFamide application. $\boldsymbol{A}$, The mean fundamental frequencies of activity in adult PD and DG neurons were not significantly different (unpaired $t$ test, $p=0.31, n=$ 5). $\boldsymbol{B}$, The mean fundamental frequency of activity in embryonic $\operatorname{lpm}(n=6)$ was significantly higher than the mean fundamental frequency of activity in embryonic $p d m(n=6)$ and dgm $(n=7)$ (one-way ANOVA, Fisher's PLSD, $p<0.0001$; Bonferroni/Dunn, $p<0.0001)$. Significant difference, ${ }^{* * *} p<0.001$. The mean fundamental frequencies of activity in embryonic $\mathrm{pdm}$ and dgm were not significantly different (unpaired $t$ test, $p=0.1$ ). Preparations in which there was silent or sparsely bursting in dgm (3 of 10 preparations) were not included in these graphs and calculations. $C$, Phase of adult DG $(n=6)$ neurons in relation to phase of adult PD neurons. DG activity ( $y$-axis) is plotted as position in the PD phase on the $x$-axis (PD phase $=1$ ). $D$, Phase of embryonic Ipm $(n=3)$ and dgm $(n=4)$ neurons in the pdm phase.

in the firing patterns of neurons relative to each other. Thus, to estimate phase relationships we first determined the frequency most common to two neurons, using PD as the reference for LP and DG (see Materials and Methods, Spectral analysis). At this peak coherent frequency, we calculated the point in the PD cycle that LP or DG fired such that activity at a phase of 0 indicates synchronous firing and a phase of 0.5 indicates alternating firing. We also estimated the degree to which the frequency of two signals is correlated, calculated as the coherence magnitude, where 0 represents no correlation and 1 represents perfect correlation at the peak coherent frequency. In $\mathrm{Val}^{1}$-SIFamide, LP neurons were not activated in the adult, and adult DG and PD neurons fired nearly simultaneously (mean DG phase in the PD phase at $\sim 0.95$, $n=6$; mean coherence magnitude. $0.93 \pm 0.09$ ) (Fig. 5C).

In $\mathrm{Val}^{1}$-SIFamide, at the peak coherent frequency between embryonic pdm and dgm (mean coherence magnitude, $0.73 \pm$ 0.2 ), activity in 4 of 6 embryonic pdm occurred nearly simultaneously with dgm (at $\sim 0.04$ in the PD phase), and in 2 of 6 preparations pdm activity occurred out of phase, firing in alternation with dgm (at $\sim 0.45$ in the PD phase) (Fig. $5 D$ ). At the peak coherent frequency between $\mathrm{lpm}$ and pdm (mean coherence magnitude $=0.83 \pm 0.08$ ), activity in embryonic lpm occurred out of phase with pdm $(0.34 \pm 0.10$ in the pdm phase, $n=3)$ (Fig. $5 D)$. The DG/PD phase relationships in embryos and adults were not significantly different (Watson-Williams test, $p=0.41, n=$ 6) (Fig. 5C,D).

\section{Direct effects of CabTRP were different in adult and embryonic STG}

CabTRP is a peptide that is present in the adult STG inputs but is not present in the embryonic STG inputs (Fénelon et al., 1999; Cape et al., 2008). Previous studies have shown that CabTRP activates a subset of adult pyloric neurons after neuromodulatory inputs are removed (Thirumalai and Marder, 2002), but the effects of CabTRP on adult gastric mill neurons under these conditions have not been determined. Therefore, we bath-applied CabTRP to adult STG after neuromodulatory inputs were removed while recording from the PD, LP, and DG neurons. As previously described, adult LP and DG neurons were silent when the modulatory inputs were removed, and the adult PD neuron continued to burst slowly (Fig. 6A). In $10^{-6} \mathrm{M}$ CabTRP, the LP neuron remained silent, the DG neuron began bursting, and bursting in the $\mathrm{PD}$ neuron became faster $(\sim 0.3 \mathrm{~Hz})$ and was synchronous with the bursting in the DG neuron (Fig. $6 B$ ).

A recent study showed that, when endogenous neuromodulatory inputs were present, CabTRP increased the amount of slow activity produced by embryonic gastric mill neurons, making the embryonic motor patterns appear more like those in the adult (Rehm et al., 2008). However, these experiments did not show that these effects were mediated by CabTRP receptors in the STG itself. Thus, we asked whether CabTRP has direct modulatory effects on the embryonic STG isolated from its neuromodulatory inputs. When isolated from their neuromodulatory inputs, all embryonic STG neurons were silent (Fig. 6C). In $10^{-6} \mathrm{M}$ CabTRP, all embryonic neurons became active: during this stretch of the recording, $\mathrm{lpm}$ and pdm were active at different frequencies, and one $\sim 2.5$ s period of activity was observed in dgm (Fig. 6D).

The differences between isolated adult and embryonic STG responses to CabTRP are readily seen in the spectrograms. The adult LP spectrogram is blue because LP was silent (Fig. 7A), whereas the embryonic lpm was active at $\sim 0.2 \mathrm{~Hz}$ (Fig. $8 B$ ). Adult $\mathrm{PD}$ activity was more regular and faster $(\sim 0.3 \mathrm{~Hz})($ Fig. $7 A)$ than the embryonic pdm $(\sim 0.2 \mathrm{~Hz})$ (Fig. $7 B)$. DG in the adult was active at $\sim 0.3 \mathrm{~Hz}$ (Fig. $7 A$ ), but in the embryo, dgm was silent in CabTRP except for the period of activity in the middle of the recording (Fig. $7 B$ ).

When we pooled the data from all adult preparations, we found that when neuromodulatory inputs were removed, LP neurons were silent both in control saline $(n=6)$ (data not shown) and in CabTRP $(n=6)$ (Fig. $8 A)$. In CabTRP, the mean fundamental frequency of bursting in adult PD neurons $(0.23 \pm$ $0.09 \mathrm{~Hz}, n=6$ ) (Fig. $8 \mathrm{~A}$ ) was not significantly faster than the mean fundamental frequency of activity in control saline $(0.15 \pm$ $0.1 \mathrm{~Hz}, n=6$, data not shown; paired $t$ test, $p=0.23, n=6)$. CabTRP elicited bursting in adult DG neurons at a mean fundamental frequency $(0.24 \pm 0.08 \mathrm{~Hz}, n=6)$ (Fig. $8 A$ ), which was not significantly different from the mean fundamental frequency of DG activity in control saline $(0.22 \pm 0.27 \mathrm{~Hz}, n=6$, data not shown; paired $t$ test, $p=0.84, n=6)$, or PD activity in CabTRP (unpaired $t$ test, $p=0.89, n=6$ ) (Fig. $8 A$ ).

In all embryonic preparations, lpm, pdm, and dgm were silent in control saline when the neuromodulatory inputs were removed (data not shown). In CabTRP, the mean fundamental frequencies of activity in pdm $(0.23 \pm 0.08 \mathrm{~Hz}, n=7)$ and $\mathrm{lpm}$ $(0.23 \pm 0.07 \mathrm{~Hz}, n=7)$ were not statistically different (unpaired $t$ test, $p=0.15, n=7$ ) (Fig. $8 B$ ). We did not calculate the fundamental frequency for embryonic dgm because activity in dgm was highly irregular (1-4 bursts in 5-10 min recordings). CabTRPactivated motor patterns were not changed when the muscles were hyperpolarized, and the waveforms of these events were typical of embryonic EJPs.

The CabTRP-activated motor patterns in embryos and adults were different. In CabTRP, lpm was activated in the embryo but the LP neuron was not activated in the adult. The embryonic dgm 
was either silent or produced very slow, infrequent activity, but in the adult, DG fired together with the PD neuron. There were no statistical differences between the mean fundamental frequencies of activity in adult PD neurons and embryonic pdm [unpaired $t$ test, $p=0.95, n=6$ (adult), $n=7$ (embryo)].

Comparing the phase relationships of the CabTRP-activated motor patterns between embryos and adults, adult PD and DG neurons fired together (DG phase at $\sim 0$ in the PD phase; mean coherence magnitude, $0.92 \pm 0.09$ ) (Fig. $8 C$ ), whereas embryonic lpm and pdm fired in alternation (lpm phase at $\sim 0.5$ in the pdm phase; mean coherence magnitude, $0.71 \pm 0.12$ ) (Fig. 8D).

\section{Direct effects of RPCH were similar in adult and embryonic STG}

We also wanted to examine the maturity of the embryonic STG response to a modulator whose expression in the embryonic inputs is mature. Red pigment concentrating hormone (RPCH) is a peptide that modulates adult pyloric neurons and has a very similar expression pattern in the adult and embryonic STNS (Fénelon et al., 1999; Thirumalai and Marder, 2002). When RPCH was bath-applied to an adult STG isolated from its neuromodulatory inputs, the activity patterns in the PD and LP neurons were similar, and the DG neuron fired bursts at a slower frequency (Fig. $9 A, B)$. In embryos, RPCH also activated all three neurons, resulting in motor pattern in which lpm and pdm were active at approximately the same frequency, which was faster than dgm (Fig. 9C,D).

Figure 10 shows the adult and embryonic motor patterns in $\mathrm{RPCH}$ during the entire recording of the experiment from Figure 9. The spectrograms of the output from the adult DG neuron (fundamental frequency, $0.04 \mathrm{~Hz}$ ) and the embryonic dgm (fundamental frequency, $0.04 \mathrm{~Hz}$ ) are similar (Fig. $10 A, B$, last panels). The adult LP and embryonic lpm spectrograms also look similar (most activity occurred less than $\sim 0.04 \mathrm{~Hz}$ ) but, the fundamental frequency of activity in this adult LP neuron $(0.13$ $\mathrm{Hz}$ ) was faster than that in embryonic lpm $(0.08 \mathrm{~Hz})$ (Fig. 10A, $B$, center panels). Activity in the spectrogram of the adult PD neuron (fundamental frequency $=0.09 \mathrm{~Hz}$ ) was faster than that in embryonic pdm $(0.05 \mathrm{~Hz})$ (Fig. $10 \mathrm{~A}, B$, first panels).

When we calculated the mean fundamental frequencies of activity in adults, we found that LP neurons were silent in control saline (data not shown), but were active in RPCH at $0.17 \pm 0.09$ $\mathrm{Hz}(n=7)$ (Fig. 11A). RPCH did not significantly change the mean fundamental frequency of activity in adult PD neurons compared with control saline (mean fundamental frequencies: in control saline, $0.13 \pm 0.05 \mathrm{~Hz}, n=7$; in $\mathrm{RPCH}, 0.19 \pm 0.11 \mathrm{~Hz}$, $n=7$; paired $t$ test, $p=0.17, n=7$ ) (Fig. $11 A$, control saline data not shown). In RPCH, DG neurons produced bursts at a mean fundamental frequency $(0.04 \pm 0.01 \mathrm{~Hz}, n=7)$ that was significantly slower than that of DG neurons in control saline $(0.83 \pm$ $0.53 \mathrm{~Hz}, n=7$; paired $t$ test, $p=0.01, n=7$ ) (Fig. $11 A$, control data not shown). In RPCH, the mean fundamental frequencies of activity in LP and PD were not significantly different (one-way ANOVA, $p=0.64, n=7$ ), but the mean frequencies of activity in both LP and PD neurons were faster than DG neurons (one-way ANOVA, $p=0.004, n=7$ ) (Fig. $11 A$ ).

In control saline, all embryonic neurons were silent after neuromodulatory inputs were removed. In $\mathrm{RPCH}$, the mean fundamental frequencies of the activity in $\operatorname{lpm}(0.10 \pm 0.03 \mathrm{~Hz}, n=7)$ and $\operatorname{pdm}(0.11 \pm 0.04 \mathrm{~Hz}, n=7)$ were not statistically different (one-way ANOVA, $p=0.80, n=7$ ), and the mean fundamental 
Table 1. Summary of neuromodulator expression, receptor presence, and modulatory function in the development of the lobster STG

\begin{tabular}{llll}
\hline & $\begin{array}{l}\text { Neuromodulator expression } \\
\text { mature in late embryonic STNS }\end{array}$ & $\begin{array}{l}\text { Neuromodulator receptors } \\
\text { present in late embryonic STG }\end{array}$ & $\begin{array}{l}\text { Neuromodulator response } \\
\text { mature in late embryonic STG }\end{array}$ \\
\hline RPCH & Yes & Yes & Yes \\
CabTRP & No & Yes & No \\
Val'-SIFamide & No & Yes & No \\
Proctolin & Yes & Yes & Unknown \\
AST-3 & Yes & Yes & Unknown \\
5-HT & Yes & Yes & Unknown \\
DA & Yes & Yes & Unknown \\
CCAP & Yes & Yes & Unknown \\
GABA & No & Yes & Unknown \\
HA & Yes & Yes & Unknown \\
\hline
\end{tabular}

In the embryonic STNS, the expression of all neuromodulators, except CabTRP, Val' ${ }^{1}$-SIFamide, and GABA, is mature by the end of embryonic development (Fénelon et al., 1999; Kilman et al., 1999; Pulver et al., 2003; Ducret et al., 2007; Cape et al., 2008). The receptors for all neuromodulators are present, given by the activation of at least one neuron in the isolated embryonic STG. Receptor presence of DA, CCAP, GABA, and HA were demonstrated by previous experiments (Richards and Marder, 2000; Pulver et al., 2003; Ducret et al., 2007). Simultaneous recordings from at least three neurons were used to determine the maturity of the neuromodulator response in isolated embryonic STG. RPCH had similar physiological effects in embryonic and adult STG (Fig. 11). Val ${ }^{1}$-SIFamide and CabTRP had different physiological effects in embryos compared with adults (Figs. 5, 8). between adult LP/PD, $0.95 \pm 0.04$; mean coherence magnitude between embryonic $\mathrm{lpm} / \mathrm{pdm}=0.76 \pm 0.13$ ) and was not significantly different across development (Watson-Williams test, $p=0.86, n=7$ ) (Fig. 11C,D). The phase relationship of the output from DG and PD was significantly different between embryos and adults (Watson-Williams test, $p=0.002, n=7$; mean coherence magnitude between adult DG/PD, $0.79 \pm 0.13$; mean coherence magnitude between embryonic dgm/pdm, $0.68 \pm 0.15)$. The phase relationships of DG and PD activity were more variable across experiments, particularly in the embryo, where dgm activity could occur at many points in the pdm phase.

\section{Other neuromodulator receptors in the embryonic STG}
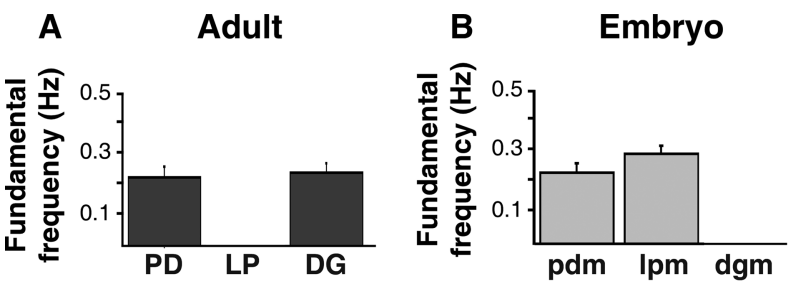

C

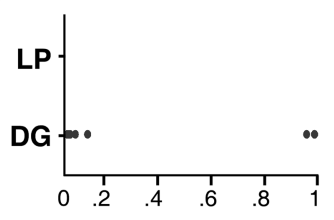

Phase of coherent activity in PD time in pdm time
D

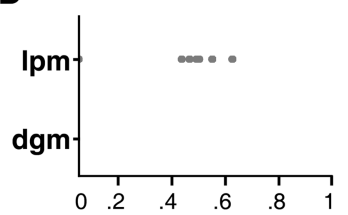

Phase of coherent activity
Figure 8. Summary of frequency and phase relationships of adult and embryonic CabTRPactivated motor patterns. $\boldsymbol{A}-\boldsymbol{D}$, Output from adult $(\boldsymbol{A}, \boldsymbol{C})$ and embryonic $(\boldsymbol{B}, \boldsymbol{D})$ neurons during $10^{-6} \mathrm{M}$ CabTRP application. $\boldsymbol{A}$, The mean fundamental frequencies of activity in adult PD and $D G$ neurons were not significantly different (unpaired $t$ test, $p=0.89, n=6$ ). $\boldsymbol{B}$, The mean fundamental frequencies of activity in embryonic Ipm and pdm were not significantly different (unpaired $t$ test, $p=0.15, n=7$ ). Embryonic dgm produced $1-4$ bursts in 6 of 7 preparations and was silent in one preparation; therefore, DG activity was not quantified. C, Phase relationships of adult DG and PD neurons $(n=6)$. $D$, Phase relationships of embryonic lpm and pdm $(n=7)$.

frequencies of activity in both $\mathrm{lpm}$ and pdm were faster than dgm $(0.04 \pm 0.14 \mathrm{~Hz}, n=7)$ (one-way ANOVA, $p=0.001, n=7$ ) (Fig. $11 B$ ). RPCH-induced events were typical of EJPs.

When we compared the fundamental frequencies of the output from embryonic and adult neurons in $\mathrm{RPCH}$, we found that LP and PD activity was faster in adults compared with embryos, however this difference was not statistically significant (unpaired $t$ test, LP/lpm, $p=0.089, n=7 ; \mathrm{PD} / \mathrm{pdm}, p=0.09, n=7$ ). The mean fundamental frequencies of activity in adult DG and embryonic dgm were similar (unpaired $t$ test, $p=0.52, n=7$ ).

In RPCH, the phase relationships of activity in embryonic and adult STG neurons were similar, although not identical. In adults and embryos, at their peak coherent frequency, the output from LP and PD neurons tended to alternate with each other (between 0.4 and 0.6 in the PD phase; mean coherence magnitude
Next we asked how many of the receptors to the final adult neuromodulator complement are present in the embryo. Previous work had shown that the isolated embryonic STG responds to dopamine (DA), crustacean cardioactive peptide (CCAP), GABA, and histamine (HA) (Richards and Marder, 2000; Pulver et al., 2003; Ducret et al., 2007) (Table 1). Although we did not do a detailed comparison with adult preparations, we tested for the presence of receptors for proctolin and allatostatin type A 3 (AST-3), modulators that are both present in the late embryonic STG inputs, and 5-HT, which first appears in the STG inputs during mid-late larval stages (Fénelon et al., 1999; Kilman et al., 1999). When bath-applied to the isolated embryonic STG, $10^{-6}$ M proctolin $(n=7)$ and $10^{-4} \mathrm{M} 5$-HT $(n=9)$ produced output from embryonic pyloric neurons, and in five preparations $10^{-6} \mathrm{M}$ AST-3 silenced bursting in lpm when reactivated with other neuromodulators (data not shown). In addition to rhythmic events typical of EJPs, both proctolin $(n=5)$ and 5-HT $(n=2)$ also appeared to activate some muscle action potentials, as evidenced by depolarizations with irregular and slow waveforms.

Using results from the present study and previous studies (Richards and Marder, 2000; Pulver et al., 2003; Ducret et al., 2007), we have summarized the maturity of neuromodulator expression in the late embryonic STNS, the neuromodulator receptor presence in the embryonic STG, and, when possible, compared the neuromodulator receptor function in embryonic and adult STG (Table 1). Receptors for many neuromodulators (in fact, receptors for all modulators tested thus far) are present by the end of embryonic development.

\section{Discussion}

In a variety of developing neuronal networks, many aspects of the circuit and its inputs change as the output matures (Sernagor et al., 2001; Montoro and Yuste, 2004; Moody and Bosma, 2005; Kiehn, 2006; Sillar et al., 2008). It is unknown, however, which changes best account for the maturation of the output from the circuit. Although the effects of one or two neuromodulators have been studied in other developing systems (Rajaofetra et al., 1989; Ziskind-Conhaim et al., 1993; Sillar et al., 1995; Pflüger, 1999; Vinay et al., 2000; Drapeau et al., 2002; Pearson et al., 2003; Simakajornboon and Kuptanon, 2005; Rauscent et al., 2006; Parker and Gilbey, 2007), to the best of our knowledge this study produces the most comprehensive summary to date of the embryonic effects of multiple modulators that are active in the adult 
(Table 1). Our findings suggest that, although many neuromodulator receptors are already present on the neurons in the embryonic STG circuit, the responses of the circuit to some neuromodulators, particularly those with developmental expression differences, are immature.

\section{Direct effects of RPCH may indicate that the embryonic STG is mostly mature}

Previous anatomical studies have shown that the neuropeptide RPCH is localized similarly in embryonic and adult neuromodulatory inputs (Fénelon et al., 1999), and that RPCH has modulatory effects on a subset of pyloric neurons in the adult STG (Thirumalai and Marder, 2002). We found that RPCH produced similar motor patterns in isolated adult and embryonic STG. These results are potentially consistent with the interpretation that the basic configuration of the STG network is mature by the end of embryonic stage, as was argued by Le Feuvre et al. (1999). However, it should also be noted that recent theoretical results have demonstrated that similar circuit output can be produced by different underlying mechanisms (Prinz et al., 2004; Marder and Goaillard, 2006). Consequently, although it is useful to compare the responses of embryonic and adult STG to the same modulator, it must be remembered that similar responses, such as those seen in RPCH, do not necessarily imply the existence of identical circuit mechanisms.

\section{$\mathrm{Val}^{1}$-SIFamide distribution in the embryonic STNS}

Using MALDI mass spectrometry, it has recently been shown that, in contrast to the adult, $\mathrm{Val}^{1}$-SIFamide is the most abundant peptide in the embryonic STG inputs (Cape et al., 2008). Although the $\mathrm{Val}^{1}$-SIFamide distribution was similar in adult and embryonic STG, Val ${ }^{1}$-SIFamide was absent from many regions of the embryonic neuromodulatory inputs, and there were very few changes in the distribution of $\mathrm{Val}^{1}{ }^{1}$-SIFamide during larval development. If $\mathrm{Val}^{1}$-SIFamide localization becomes adult-like when the STNS output becomes mature, after larval development (Casasnovas and Meyrand, 1995), Val ${ }^{1}$ SIFamide could be important to the functional maturation of the STNS.

\section{Developmental regulation of the modulatory responses of CabTRP and Val $^{1}{ }^{-}$-SIFamide}

CabTRP and $\mathrm{Val}^{1}$-SIFamide both elicited motor patterns in the isolated embryonic STG, demonstrating the presence of $\mathrm{Val}^{1}{ }^{-}$-SIFamide and CabTRP receptors in the embryonic STG. Both CabTRP and $\mathrm{Val}^{1}{ }^{1}$-SIFamide elicited different motor patterns in embryos and adults, and these differences can contrib-
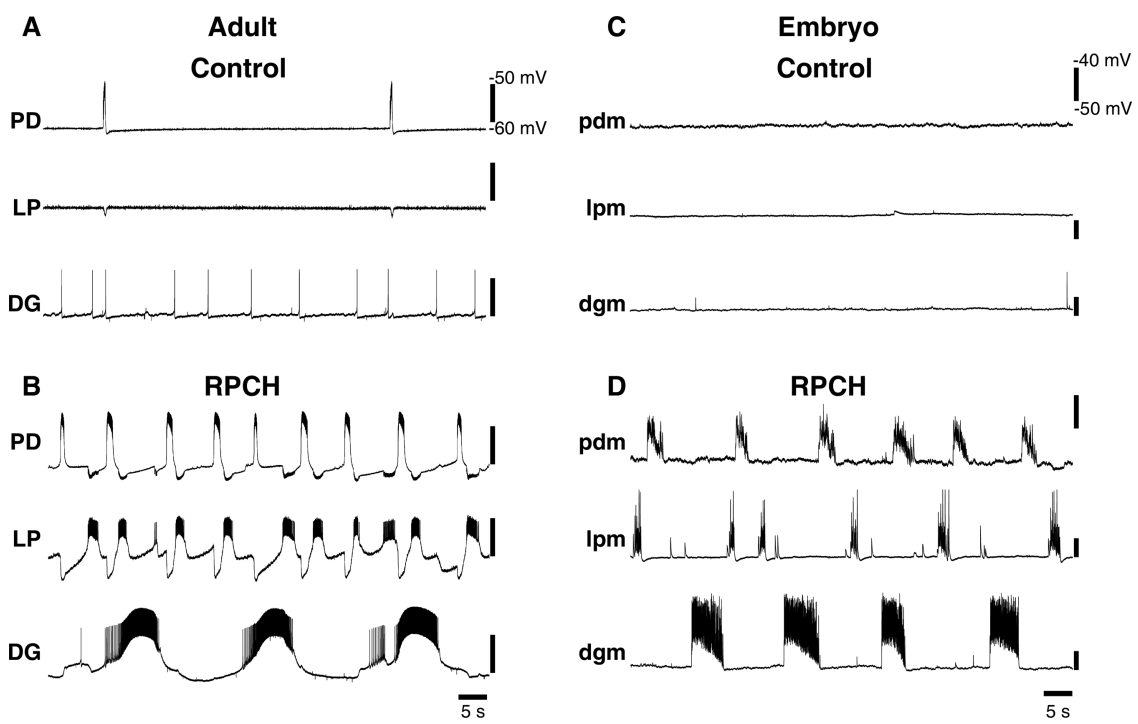

RPCH effects on an isolated adult and embryonic STG. $\boldsymbol{A}$, Adult, Simultaneous intracellular recordings from PD, LP, Simultaneous intracellular recordings from pdm, Ipm, and dgm, neuromodulatory inputs removed, in control saline. $\boldsymbol{D}$, Same

A

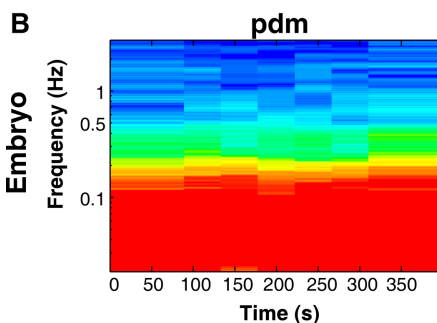

Figure 10. Spectrograms of adult and embryonic RPCH-activated motor patterns. $\boldsymbol{A}$, Adult PD, LP and DG spectrograms, total recording time in $10^{-6} \mathrm{M} R P C H$ from Figure $9 B$. $B$, Embryonic pdm, Ipm, and dgm spectrograms, total recording time in $10^{-6} \mathrm{M}$ $\mathrm{RPCH}$, preparation shown in Figure 9D.

ute to the immaturity of the embryonic motor patterns when neuromodulatory inputs are intact. The developmental differences in CabTRP and $\mathrm{Val}^{1}$-SIFamide-activated motor patterns in the isolated STG could indicate differences in the relative number and/or distribution of CabTRP and Val ${ }^{1}$ SIFamide receptors in the embryo and the adult. Furthermore, it is also possible that CabTRP and $\mathrm{Val}^{1}$-SIFamide receptor properties change over development, with different subtypes or associated intracellular cascades present in embryos and adults.

The salient difference between embryonic and adult motor patterns in $\mathrm{Val}^{1}$-SIFamide was a lack of activation of the adult LP neurons, whereas the LP neurons were activated in embryos. One possibility is that the LP neuron in the embryo displays $\mathrm{Val}^{1}{ }^{1}$ 

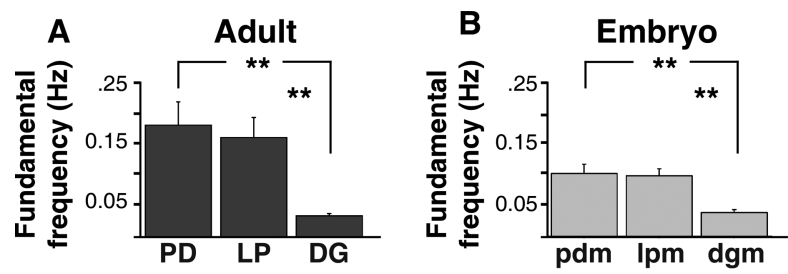

C

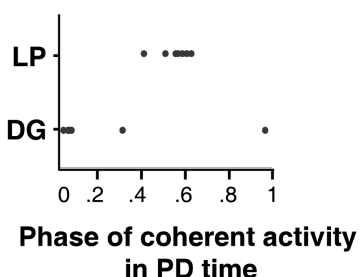

D

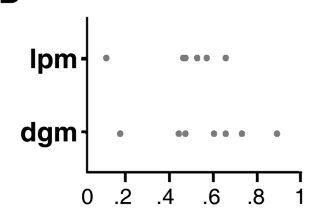

Phase of coherent activity in pdm time

Figure 11. Summary of frequency and phase relationships of adult and embryonic $\mathrm{RPCH}$-activated motor patterns. $\boldsymbol{A}-\boldsymbol{D}$, Output from adult $(\boldsymbol{A}, \boldsymbol{C})$ and embryonic $(\boldsymbol{B}, \boldsymbol{D})$ neurons during $10^{-6} \mathrm{M} \mathrm{RPCH}$ application. $\boldsymbol{A}$, The mean fundamental frequencies of activity in adult PD and LP neurons were not significantly different (one-way ANOVA, $p=0.64$, $n=7$ ), but both were faster than the mean fundamental frequency of activity in adult DG neurons (one-way ANOVA, $p=0.004, n=7$ ). Significant difference, ${ }^{* *} p<0.01$. $\boldsymbol{B}$, The mean fundamental frequencies of activity in embryonic lpm and pdm were not statistically different (one-way ANOVA, $p=0.80, n=7$ ), but both were both faster than the mean fundamental frequency of activity in embryonic dgm (one-way ANOVA, $p=0.001$, $n=7)$. C, Phase relationships of activity in adult $L P(n=7)$ and $D G(n=7)$ neurons in the PD phase. $\boldsymbol{D}$, Phase relationships of activity in embryonic $\operatorname{Ipm}(n=7)$ and dgm $(n=7)$ neurons in the pdm phase.

SIFamide receptors that are preferentially lost as the animal matures. Alternatively, stronger electrical coupling could account for the LP neuron activation even if it does not actually express $\mathrm{Val}^{1}$-SIFamide receptors.

Recent experiments have shown that CabTRP has similar, although not identical modulatory effects on motor patterns in embryos and adults when neuromodulatory inputs are intact (Rehm et al., 2008). In the adult STG, CabTRP activates a different subset of pyloric neurons than RPCH (Thirumalai and Marder, 2002). CabTRP does not appear in the inputs to the STG itself until late larval stages (Fénelon et al., 1999; Cape et al., 2008). We have shown here that the modulatory function of CabTRP is dramatically different in embryonic and adult STG. As with $\mathrm{Val}^{1}$-SIFamide, LP was activated by CabTRP in embryonic but not adult STG. Furthermore, in CabTRP, PD and DG neurons fired together in adults, but the embryonic DG neuron produced rare "bursts" of activity. It is interesting that in adults, DG and PD neurons fired together when re-activated by all three neuromodulatory peptides, CabTRP, Val ${ }^{1}$-SIFamide, and RPCH. It is possible that receptors for these peptides are downregulated during development, and are absent in adult DG neurons, allowing the DG neuron to fire with the PD neuron if they are weakly electrically coupled (A. E. Tobin, personal communication) (Ducret et al., 2006)

The adult STG is modulated by a large number of substances (Marder and Bucher, 2007). It is interesting that the embryonic STG network responds to all substances tested thus far, and that the responses to CabTRP and 5-HT precede their arrival in the neuromodulatory inputs (Fénelon et al., 1999; Kilman et al., 1999; Cape et al., 2008). Furthermore, neuromodulators with immature expression patterns in the embryo have different physiological effects on embryonic and adult STG networks. A similar phenomenon has been observed for

nitric oxide, 5-HT, and substance $\mathrm{P}$ in lamprey, frog, and rat spinal cord networks (Rajaofetra et al., 1989; ZiskindConhaim et al., 1993; Vinay et al., 2000; Rauscent et al., 2006; Parker and Gilbey, 2007).

Serotonin and tachykinin-related peptides are thought to influence development in a wide variety of invertebrate and vertebrate nervous systems (Pagliardini et al., 2003; Satake et al., 2003; Casini et al., 2004; Wong-Riley and Liu, 2005; Catalani et al., 2006; Greer et al., 2006; Rauscent et al., 2006; Vitalis et al., 2007). In mammalian and Drosophila CNS, the distribution of tachykinin-like peptide receptors is more widespread earlier in development, and is refined during postnatal development (Quirion and Dam, 1986; Li et al., 1991; Monnier et al., 1992; Kar and Quirion, 1995; Aita et al., 2005; Catalani et al., 2006), perhaps to support an early trophic role for tachykinin-like peptides, like CabTRP.

Our physiological studies are consistent with the possibility that there is a wider distribution of CabTRP and $\mathrm{Val}^{1}{ }^{1}$ SIFamide receptors in the embryonic STG, but do not establish it because of the confound of widespread electrical coupling. Perhaps early promiscuous receptor expression supports a developmental, trophic role for CabTRP, which is present in the embryonic hemolymph (Pulver and Marder, 2002), and $\mathrm{Val}^{1}$-SIFamide, which is present in greater relative abundance in the embryonic STG compared with the adult (Cape et al., 2008). Furthermore, neurotransmitters and neuromodulators can regulate the maturation of their own effects (Ganguly et al., 2001; Colonnese et al., 2003; Leitch et al., 2005). Thus, perhaps $\mathrm{Val}^{1}$-SIFamide levels are elevated in the embryonic STG inputs to mature the $\mathrm{Val}^{1}$-SIFamide response, by refining the localization of $\mathrm{Val}^{1}$-SIFamide receptors. And perhaps CabTRP has not yet arrived in sufficient quantities to prune the expression of CabTRP receptors in the embryonic STG.

\section{Conclusions}

Our data are consistent with a model of circuit maturation in which the fundamental architecture matures before its output, although considerable fine-tuning must continue. It is interesting to note that many neuromodulator receptors are seen early, before the network output is mature, and before some of the neuromodulatory substances are present in the inputs to the circuit. Furthermore, the responses of modulators with immature expression patterns are also immature, suggesting that the final functional maturation of the output of the circuit may depend on both the maturation of the neuromodulatory inputs and subtle changes in receptor number and distribution.

\section{References}

Aita M, Seo K, Fujiwara N, Takagi R, Maeda T (2005) Postnatal changes in the spatial distributions of substance $\mathrm{P}$ and neurokinin-1 receptor in the trigeminal subnucleus caudalis of mice. Brain Res Dev Brain Res 155:33-41.

Bucher D, Taylor AL, Marder E (2006) Central pattern generating neurons simultaneously express fast and slow rhythmic activities in the stomatogastric ganglion. J Neurophysiol 95:3617-3632.

Bucher D, Johnson CD, Marder E (2007) Neuronal morphology and neuropil structure in the stomatogastric ganglion of the lobster, Homarus americanus. J Comp Neurol 501:185-205.

Cape SS, Rehm KJ, Ma M, Marder E, Li L (2008) Mass spectral comparison of the neuropeptide complement of the stomatogastric ganglion and brain in the adult and embryonic lobster, Homarus americanus. J Neurochem 105:690-702.

Casasnovas B, Meyrand P (1995) Functional differentiation of adult 
neural circuits from a single embryonic network. J Neurosci 15:5703-5718.

Casini G, Dal Monte M, Fornai F, Bosco L, Willems D, Yang Q, Zhou ZJ, Bagnoli P (2004) Neurokinin 1 receptor expression and substance P physiological actions are developmentally regulated in the rabbit retina. Neuroscience 124:147-160.

Catalani E, Dal Monte M, Gangitano C, Lucattelli M, Fineschi S, Bosco L, Bagnoli P, Casini G (2006) Expression of substance P, neurokinin 1 receptors (NK1) and neurokinin 3 receptors in the developing mouse retina and in the retina of NK1 knockout mice. Neuroscience 138:487-499.

Christie AE, Stemmler EA, Peguero B, Messinger DI, Provencher HL, Scheerlinck P, Hsu YW, Guiney ME, de la Iglesia HO, Dickinson PS (2006) Identification, physiological actions, and distribution of VYRKPPFNGSIFamide $\left(\mathrm{Val}^{1}\right)$-SIFamide) in the stomatogastric nervous system of the American lobster Homarus americanus. J Comp Neurol 496:406-421.

Colonnese MT, Shi J, Constantine-Paton M (2003) Chronic NMDA receptor blockade from birth delays the maturation of NMDA currents, but does not affect AMPA/kainate currents. J Neurophysiol 89: $57-68$.

Demas J, Eglen SJ, Wong RO (2003) Developmental loss of synchronous spontaneous activity in the mouse retina is independent of visual experience. J Neurosci 23:2851-2860.

Drapeau P, Saint-Amant L, Buss RR, Chong M, McDearmid JR, Brustein E (2002) Development of the locomotor network in zebrafish. Prog Neurobiol 68:85-111.

Ducret E, Alexopoulos H, Le Feuvre Y, Davies JA, Meyrand P, Bacon JP, Fénelon VS (2006) Innexins in the lobster stomatogastric nervous system: cloning, phylogenetic analysis, developmental changes and expression within adult identified dye and electrically coupled neurons. Eur J Neurosci 24:3119-3133.

Ducret E, Le Feuvre Y, Meyrand P, Fénelon VS (2007) Removal of GABA within adult modulatory systems alters electrical coupling and allows expression of an embryonic-like network. J Neurosci 27:3626-3638.

Feller MB (1999) Spontaneous correlated activity in developing neural circuits. Neuron 22:653-656.

Fénelon VS, Casasnovas B, Faumont S, Meyrand P (1998) Ontogenetic alteration in peptidergic expression within a stable neuronal population in lobster stomatogastric nervous system. J Comp Neurol 399:289-305.

Fénelon VS, Kilman V, Meyrand P, Marder E (1999) Sequential developmental acquisition of neuromodulatory inputs to a central patterngenerating network. J Comp Neurol 408:335-351.

Ganguly K, Schinder AF, Wong ST, Poo M (2001) GABA itself promotes the developmental switch of neuronal GABAergic responses from excitation to inhibition. Cell 105:521-532.

Greer JJ, Funk GD, Ballanyi K (2006) Preparing for the first breath: prenatal maturation of respiratory neural control. J Physiol 570:437-444.

Helluy SM, Beltz BS (1991) Embryonic development of the American lobster (Homarus americanus): quantitative staging and characterization of an embryonic molt cycle. Biol Bull 180:355-371.

Kar S, Quirion R (1995) Neuropeptide receptors in developing and adult rat spinal cord: an in vitro quantitative autoradiography study of calcitonin gene-related peptide, neurokinins, mu-opioid, galanin, somatostatin, neurotensin and vasoactive intestinal polypeptide receptors. J Comp Neurol 354:253-281.

Kiehn O (2006) Locomotor circuits in the mammalian spinal cord. Annu Rev Neurosci 29:279-306.

Kilman V, Fénelon VS, Richards KS, Thirumalai V, Meyrand P, Marder E (1999) Sequential developmental acquisition of cotransmitters in identified sensory neurons of the stomatogastric nervous system of the lobsters, Homarus americanus and Homarus gammarus. J Comp Neurol 408:318-334.

Le Feuvre Y, Fénelon VS, Meyrand P (1999) Central inputs mask multiple adult neural networks within a single embryonic network. Nature 402:660-664.

Leitch E, Coaker J, Young C, Mehta V, Sernagor E (2005) GABA type-A activity controls its own developmental polarity switch in the maturing retina. J Neurosci 25:4801-4805.

Li XJ, Wolfgang W, Wu YN, North RA, Forte M (1991) Cloning, heterolo- gous expression and developmental regulation of a Drosophila receptor for tachykinin-like peptides. EMBO J 10:3221-3229.

Marder E, Bucher D (2007) Understanding circuit dynamics using the stomatogastric nervous system of lobsters and crabs. Annu Rev Physiol 69:291-316.

Marder E, Goaillard JM (2006) Variability, compensation and homeostasis in neuron and network function. Nat Rev Neurosci 7:563-574.

Monnier D, Colas JF, Rosay P, Hen R, Borrelli E, Maroteaux L (1992) NKD, a developmentally regulated tachykinin receptor in Drosophila. J Biol Chem 267:1298-1302.

Montoro RJ, Yuste R (2004) Gap junctions in developing neocortex: a review. Brain Res Brain Res Rev 47:216-226.

Moody WJ, Bosma MM (2005) Ion channel development, spontaneous activity, and activity-dependent development in nerve and muscle cells. Physiol Rev 85:883-941.

Nishimaru H, Kudo N (2000) Formation of the central pattern generator for locomotion in the rat and mouse. Brain Res Bull 53:661-669.

Pagliardini S, Ren J, Greer JJ (2003) Ontogeny of the pre-Bötzinger complex in perinatal rats. J Neurosci 23:9575-9584.

Parker D, Gilbey T (2007) Developmental differences in neuromodulation and synaptic properties in the lamprey spinal cord. Neuroscience 145:142-152.

Pearson SA, Mouihate A, Pittman QJ, Whelan PJ (2003) Peptidergic activa-

tion of locomotor pattern generators in the neonatal spinal cord. J Neurosci 23:10154-10163.

Percival DB, Walden AT (1993) Spectral analysis for physical applications: multitaper and conventional univariate techniques. Cambridge, UK: Cambridge UP.

Pflüger HJ (1999) Neuromodulation during motor development and behavior. Curr Opin Neurobiol 9:683-689.

Prinz AA, Bucher D, Marder E (2004) Similar network activity from disparate circuit parameters. Nat Neurosci 7:1345-1352.

Pulver SR, Marder E (2002) Neuromodulatory complement of the pericardial organs in the embryonic lobster, Homarus americanus. J Comp Neurol 451:79-90.

Pulver SR, Thirumalai V, Richards KS, Marder E (2003) Dopamine and histamine in the developing stomatogastric system of the lobster, Homarus americanus. J Comp Neurol 462:400-414.

Quirion R, Dam TV (1986) Ontogeny of substance P receptor binding sites in rat brain. J Neurosci 6:2187-2199.

Rajaofetra N, Sandillon F, Geffard M, Privat A (1989) Pre- and post-natal ontogeny of serotonergic projections to the rat spinal cord. J Neurosci Res 22:305-321.

Rauscent A, Le Ray D, Cabirol-Pol MJ, Sillar KT, Simmers J, Combes D (2006) Development and neuromodulation of spinal locomotor networks in the metamorphosing frog. J Physiol Paris 100:317-327.

Rehm KJ, Taylor AL, Pulver SR, Marder E (2008) Spectral analyses reveal the presence of adult-like activity in the embryonic stomatogastric motor patterns of the lobster, Homarus americanus. J Neurophysiol 99:3104-3122.

Ren J, Greer JJ (2003) Ontogeny of rhythmic motor patterns generated in the embryonic rat spinal cord. J Neurophysiol 89:1187-1195.

Richards KS (2000) Development and modulation of the stomatogastric motor patterns of the lobster, Homarus americanus. $\mathrm{PhD}$ thesis, Brandeis University.

Richards KS, Marder E (2000) The actions of crustacean cardioactive peptide on adult and developing stomatogastric ganglion motor patterns. J Neurobiol 44:31-44.

Richards KS, Miller WL, Marder E (1999) Maturation of lobster stomatogastric ganglion rhythmic activity. J Neurophysiol 82: 2006-2009.

Satake H, Kawada T, Nomoto K, Minakata H (2003) Insight into tachykinin-related peptides, their receptors, and invertebrate tachykinins: a review. Zoolog Sci 20:533-549.

Sernagor E, Eglen SJ, Wong RO (2001) Development of retinal ganglion cell structure and function. Prog Retin Eye Res 20:139-174.

Sillar KT, Woolston AM, Wedderburn JF (1995) Involvement of brainstem serotonergic interneurons in the development of a vertebrate spinal locomotor circuit. Proc Biol Sci 259:65-70.

Sillar KT, Combes D, Ramanathan S, Molinari M, Simmers J (2008) 
Neuromodulation and developmental plasticity in the locomotor system of anuran amphibians during metamorphosis. Brain Res Rev 57:94-102.

Simakajornboon N, Kuptanon T (2005) Maturational changes in neuromodulation of central pathways underlying hypoxic ventilatory response. Respir Physiol Neurobiol 149:273-286.

Thirumalai V, Marder E (2002) Colocalized neuropeptides activate a central pattern generator by acting on different circuit targets. J Neurosci 22:1874-1882.

Vinay L, Brocard F, Pflieger JF, Simeoni-Alias J, Clarac F (2000) Perinatal development of lumbar motoneurons and their inputs in the rat. Brain Res Bull 53:635-647.

Vitalis T, Cases O, Passemard S, Callebert J, Parnavelas JG (2007) Embryonic depletion of serotonin affects cortical development. Eur J Neurosci 26:331-344.
Warland DK, Huberman AD, Chalupa LM (2006) Dynamics of spontaneous activity in the fetal macaque retina during development of retinogeniculate pathways. J Neurosci 26:5190-5197.

Wong RO, Oakley DM (1996) Changing patterns of spontaneous bursting activity of on and off retinal ganglion cells during development. Neuron 16:1087-1095.

Wong RO, Meister M, Shatz CJ (1993) Transient period of correlated bursting activity during development of the mammalian retina. Neuron 11:923-938.

Wong-Riley MT, Liu Q (2005) Neurochemical development of brain stem nuclei involved in the control of respiration. Respir Physiol Neurobiol 149:83-98.

Ziskind-Conhaim L, Seebach BS, Gao BX (1993) Changes in serotonininduced potentials during spinal cord development. J Neurophysiol 69: 1338-1349. 